\title{
Chondroitinsulfat bei Kniegelenksarthrose
}

\author{
Kann man mit Chondroitin wirklich \\ etwas gegen Arthrose ausrichten? \\ Eine neue Studie zeigt, dass sich un- \\ ter der Einnahme am Gelenkknorpel \\ tatsächlich etwas verändert.
}

- Kanadische Rheumatologen randomisierten 69 Patienten mit Kniegelenk-arthrosen und Synovitis in zwei Gruppen. Sie erhielten sechs Monate lang entweder $800 \mathrm{mg}$ Chondroitinsulfat täglich oder Placebo. Danach nahmen beide Gruppen für weitere sechs Monate das Verumpräparat ein. Mittels MRI wurde das Knorpelvolumen in Kniegelenk bestimmt. Die Auswertungen zeigen, dass nach sechs Monaten der Knorpelverlust in der Verumgruppe signifikant geringer ausfiel als in der Placebogruppe. Nach weiteren sechs Monaten war der Verlust persistent rückläufig. Die Symptomatik blieb allerdings unbeeinflusst.

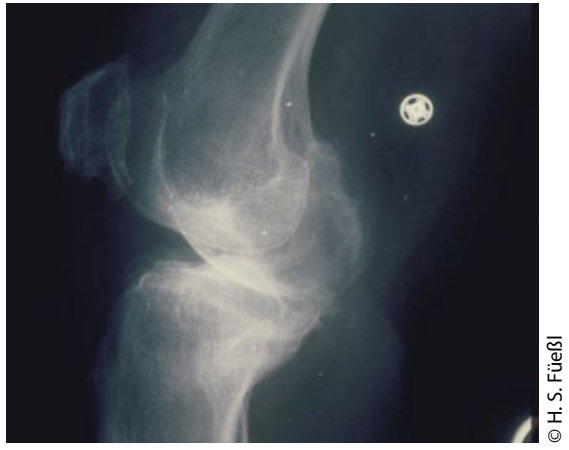

Gonarthrose: Knorpelverlust aufgehalten.

\section{Kommentar}

Die Evidenz zu Chondroitin ist voller Widersprüche. Es existieren Studien, die positive Effekte zeigen, und solche, die das nicht tun. Die vorliegende, gut gemachte Untersuchung könnte diese Diskrepanzen zumindest teilweise klären. Sie zeigt, dass objektive Parameter gut ansprechen, während die
Symptomatik diesen offenbar sehr deutlich nachhängt. Das könnte bedeuten, dass die für klinische Symptome erforderliche Therapiedauer deutlich länger ist als bislang vermutet. Zu kurz angelegte Studien würden demnach, trotz positiver Wirkungen auf den Knorpel, keine klinische Wirksamkeit erbringen. Ferner ist zu bedenken, dass das Fehlen eines positiven symptomatischen Effekts in dieser Studie auch durch die begleitende Gabe einer Rescue-Medikation bedingt sein könnte. Fazit: Chondroitin scheint zu helfen, selbst wenn der Patient davon nichts merkt.

E. ERNST :

\section{- L. M. Wildi et al. \\ Chondroitin sulphate reduces both cartilage volume loss and bone marrow lesions in knee osteoarthritis patients starting as early as 6 months after initiation of therapy: a randomi- sed, double-blind, placebo-controlled pilot study using MRI. Ann. Rheum. Dis. 2011 Mar 1. Doi: 10.1136/ard.2010.140848}

\section{Beim Haareschneiden lieber geschlossene Schuhe tragen}

\begin{abstract}
Eine Friseurin stellte sich mit einem interdigital lokalisierten Pilonidalsinus am Fuß vor. Sie trug häufig vorne offene Schuhe, wodurch frisch geschnittene Haare auf die Haut der Zehen fallen konnten. Durch die Reibung unter der Sandale drangen Haare in die Haut ein und setzten den Krankheitsprozess in Gang.
\end{abstract}

— Aus der Öffnung des Sinus pilonidalis ragten blonde, rote, braune und graue Haare von verschiedenen Kunden (siehe Abb.). Interdigital lokalisierte Sinus pilonidalis sind eine Berufserkrankung, die bei Friseuren üblicherweise nur an der Hand auftrittt. Nur wenige Fälle sind bekannt, bei denen sich die Erkrankung, so wie hier, auch am Fuß manifestierte.

Der Befund sieht auf den ersten Blick harmlos aus, die Behandlung ist jedoch langwierig und invasiv. Der betroffene Hautbezirk muss tief und umfangreich exzidiert und die Wunde lange Zeit offen behandelt werden. Daher empfiehlt es sich für Friseure, den Hautkontakt mit frisch geschnittenem Haar möglichst zu meiden.

H. S. FÜESSL =

\section{- C. C. Ekwobi}

(Department of Burns and Plastic Surgery, Wythenshawe Hospital, Manchester M23 9LT, chidi.ekwobi@uhsm.nhs.net) Brit. med. J. 341 (2010) 341: c6958.

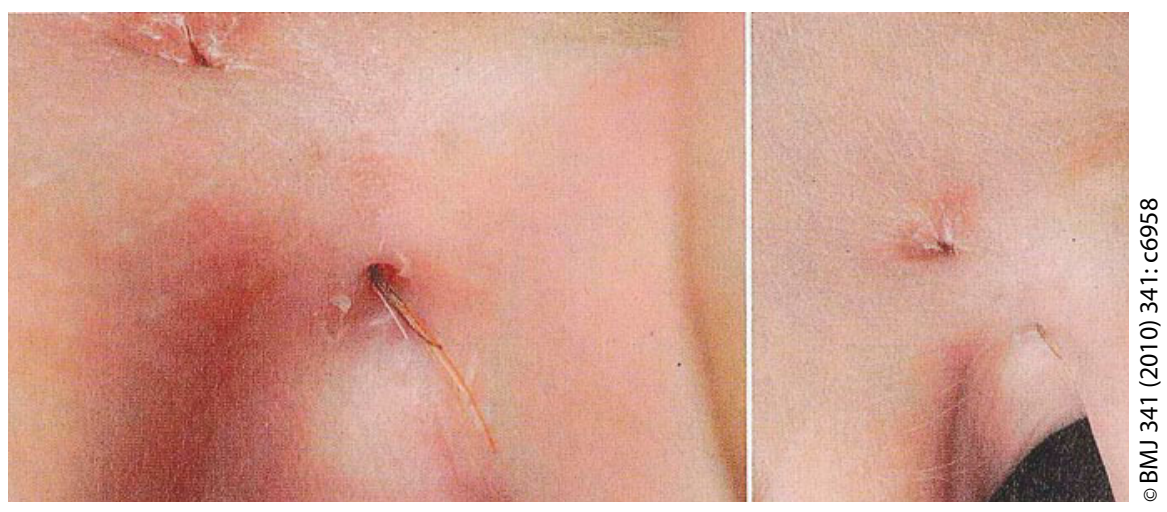

Sinus pilonidalis am Fuß bei einer Friseurin. 